\title{
Application of Multiplex Nested Methylated Specific PCR in Early Diagnosis of Epithelial Ovarian Cancer
}

\author{
Bi Wang ${ }^{1,2 \&}$, Lei Yu ${ }^{3,4 \&}$, Guo-Zhen Yang ${ }^{1 *}$, Xin Luo ${ }^{5}$, Lin Huang ${ }^{6}$
}

\begin{abstract}
Objective: To explore the application of multiplex nested methylated specific polymerase chain reaction (PCR) in the early diagnosis of epithelial ovarian carcinoma (EOC). Materials and Methods: Serum and fresh tissue samples were collected from 114 EOC patients. RUNX3, TFPI2 and OPCML served as target genes. Methylation levels of tissues were assessed by multiplex nested methylated specific PCR, the results being compared with those for carcinoma antigen 125 (CA125). Results: The serum free deoxyribose nucleic acid (DNA) methylation spectrum of EOC patients was completely contained in the DNA spectrum of cancer tissues, providing an accurate reflection of tumor DNA methylation conditions. Serum levels of CA125 and free DNA methylation in the EOC group were evidently higher than those in benign lesion and control groups $(p<0.05)$. Patients with early EOC had markedly lower serum CA125 than those with advanced EOC $(p<0.05)$, but there was no significant difference in free DNA methylation $(p>0.05)$. The sensitivity, specificity and positive predicative value (PPV) of multiplex nested methylated specific PCR were significantly higher for detection of all patients and those with early EOC than those for CA125 $(p<0.05)$. In the detection of patients with advanced EOC, the PPV of CA125 detection was obviously lower than that of multiplex nested methylated specific PCR $(p>0.05)$, but there was no significant difference in sensitivity $(p>0.05)$. Conclusions: Serum free DNA methylation can be used as a biological marker for EOC and multiplex nested methylated specific PCR should be considered for early diagnosis since it can accurately determine tumor methylation conditions.
\end{abstract}

Keywords: DNA methylation - multiplex PCR - nested PCR - epithelial ovarian cancer - early diagnosis

Asian Pac J Cancer Prev, 16 (7), 3003-3007

\section{Introduction}

Ovarian carcinoma is one of the malignant tumors commonly seen in women with mortality being $63.08 \%$, ranking the first of malignant tumors in female reproductive system in China (Wang et al., 2014). It accounts for 3\% of all female cancer-deaths, in which above $90 \%$ is caused by epithelial ovarian carcinoma (EOC) (Siegel et al., 2013). EOC is consisted of tumors with a series of biological behaviors and different pathological patterns, which can be divided into low-level carcinomas and highlevel carcinomas (Shih et al., 2004). The former, which include low-level clear cell carcinoma (CCC), low-level serous carcinoma, low-level endometrioid carcinoma and low-level mucinous carcinoma, etc., have distinctive genetic features in different pathological patterns and are slow in clinical development and favorable in prognosis. Whereas the latter contains carcinosarcoma, high-level endometrioid carcinoma, high-level serous carcinoma and undifferentiated carcinoma, etc., most of which are acute precancerous lesion with rapid development and poor prognosis. There is no simple and effective diagnostic method that can be routinely applied for the early diagnosis of EOC in clinic due to its complex pathological and clinical molecular biological characteristics (Pothuri et al., 2010).

Carcinoma antigen 125 (CA125) is a commonly used marker for ovarian carcinoma at present, but its sensitivity and specificity are low. Only $50 \%$ of patients with phase Iovarian carcinoma have increased CA125 level. However, other diseases like endometriosis (EMT) and ovarian fibroma can also lead to the increase of CA125 level (Clarke-Pearson et al., 2009). In order to search a feasible diagnostic method with practical value in clinic for the early diagnosis of EOC, multiplex nested methylated specific polymerase chain reaction (PCR) was applied in this study to detect the methylation level of serum and tissue samples in patients with ovarian carcinoma and the results were compared with the detection with CA125, which was reported as follows. 


\section{Materials and Methods}

\section{General data}

Serum and fresh tissue samples from 114 patients with ovarian carcinoma admitted in Affiliated Hospital of Guiyang Medical College from June, 2011 to December, 2014 were collected. All patients were definitely diagnosed by histopathology and (or) cytology and had complete clinical data. There were 43 with benign ovarian carcinoma (benign cancer group), aged 18 52 years with average age being $(33.95 \pm 8.75)$ years, in whom 11 were with serious cystadenoma, 10 with teratoma, 9 with ovarian endometrial cyst, 6 with mucinous cystadenoma and 7 with other diseases. And another 71 patients were with EOC (EOC group), aged 28 67 years with average age being $(54.06 \pm 9.62)$ years; 41 were with serous carcinoma, 8 with endometrioid carcinoma, 7 with CCC, 7 with mucinous carcinoma and 8 with other kind of malignant tumors; and 33 were in phase I of Federation Internationale Of Gynecologie And Obstetrigue (FIGO), 6 in phase II, 31 in phase III and 1 in phase IV. The serum samples from another 80 healthy women undergoing physical examination from Affiliated Hospital of Guiyang Medical College (control group), who were at the age of $18 \sim 61$ with average age of $(46.17 \pm 11.03)$ years, were collected as negative control.

\section{Methods}

Sample collection: All tissue samples should be collected within postoperative $24 \mathrm{~h}$, which were soaked in RNAlater at $4{ }^{\circ} \mathrm{C}$ overnight and then restored at $-80^{\circ} \mathrm{C}$ for utilization. The venous blood of all patients was collected before surgeries, which was centrifuged at $3500 \mathrm{rmp} / \mathrm{min}$ for $5 \mathrm{~min}$ at $4^{\circ} \mathrm{C}$, and then the supernatant was collected and restored at $-80^{\circ} \mathrm{C}$ for use.

Selection of specific methylated genes: Relevant literatures were retrieved and RUNX3, TFPI2 and OPCML were selected as the EOC specific methylated genes for sequential studies according to three standards, namely the high rate of methylation, the close association with the development and progression of EOC and the methylation as the primary mechanism for the transcriptional inactivation.

DNA extraction and identification: Tumor tissues of all patients were collected and deoxyribose nucleic acid (DNA) was extracted by QIAamp DNA Mini Kits (Germany Qiagen company), with all operations being conducted strictly according to the kit instructions. Ultraviolet Spectrometry Photometer (USP) was adopted to detect the absorbance (A). The DNA was regarded to be qualified when its concentration was $<50 \mathrm{ng} / \mu \mathrm{L}$ and A260/A280 was 1.8 2.0. The serum of all patients were collected and DNA was extracted by QIAamp DNA Mini Kits (Germany Qiagen company), with all operations being conducted strictly according to the kit instructions. PCR was performed with GAPDH as the reference gene (primer sequence: F: AGGTCGGAGTCAACGGATTTG, R: GTGATGGCATGGACTGTGGT). PCR products were identified by $2 \%$ agarose gel electrophoresis (AE), and DNA was regarded to be qualified when there were significantly visible products.
DNA methylation modification and identification: The tumor tissue DNA and serum free DNA were collected and methylation modification was conducted by QIAamp DNA Mini Kits (Germany Qiagen company), with all operations being conducted strictly according to the kit instructions. The DNA with methylation modification was collected with methylated ACTB (primer sequence: F: TGGTGATGGAGGAGGTTTAGTAAGT, R: AACCAATAAAACCTACTCCTCCCTTAA) as the reference gene, which was followed by the conduction of PCR. PCR products were identified by $2 \% \mathrm{AE}$, and DNA was regarded to be qualified when there were significantly visible products.

Multiplex nested methylated specific PCR: $20 \mu \mathrm{L}$ reaction system included DNA solution with methylation modification $1 \mu \mathrm{L}$, isometric mixture of primers RUNX3, TFPI 2 and OPCML $1 \mu \mathrm{L}, 2 \times$ MasterMix $10 \mu \mathrm{L}$ (USA ABI company), Enhancer $2 \mu \mathrm{L}$ (USAABI company) and ddH2O $6 \mu \mathrm{L}$. The reaction conditions: Outside: $95^{\circ} \mathrm{C} 5$ $\min , 95^{\circ} \mathrm{C} 30 \mathrm{~s}, 50^{\circ} \mathrm{C} 1 \mathrm{~min}, 50 \sim 60^{\circ} \mathrm{C} 0.2^{\circ} \mathrm{C} / \mathrm{s}, 60^{\circ} \mathrm{C} 10 \mathrm{~s}$, $72^{\circ} \mathrm{C} 45 \mathrm{~s}, 72^{\circ} \mathrm{C} 10 \mathrm{~min}$, for totally 25 cycles; Inside: $95^{\circ} \mathrm{C}$ $5 \mathrm{~min}, 95^{\circ} \mathrm{C} 30 \mathrm{~s}, 50^{\circ} \mathrm{C} 1 \mathrm{~min}, 50 \sim 60^{\circ} \mathrm{C} 0.2^{\circ} \mathrm{C} / \mathrm{s}, 60^{\circ} \mathrm{C} 10 \mathrm{~s}$, $72^{\circ} \mathrm{C} 45 \mathrm{~s}, 72^{\circ} \mathrm{C} 10 \mathrm{~min}$, for totally 35 cycles. After PCR reaction was ended, the products were identified by $2 \%$ AE so as to observe the results (Table 1).

Detection of serum CA125: Serum CA125 concentration was detected with chemiluminescence. CA125 quantitative assay kits were purchased from Germany Roche Co., Ltd. All operations were strictly conducted according to the kit instructions and the details were as follows: firstly, $20 \mu \mathrm{L}$ samples and biotinylated single-colony specific antibodies were incubated together with the ruthenium-marked specific single-colony antibodies so as to form the antigenantibody sandwich composites; secondly, magnetic bead granules covered with streptavidin (SA) were added and incubated, in which the composites would combine with the magnetic beads through biotin and SA; thirdly, the reaction products were sucked into measuring cells and the magnetic beads were absorbed on the surfaces of electrodes by electromagnetic interaction, whereas those did not combine with magnetic beads were removed by ProCell; and finally, certain voltage was implemented

\section{Table 1. Primers}

\begin{tabular}{cl}
\hline RUNX3 & \\
\hline Inside & M: 5'-TCGGGACGTATAATATTTTCG-3' \\
& U: 5'-TTGGGATGTATAATATTTTTGAG-3' \\
Outside & F: 5'-GGTTAGGGGTTTTTTAATTTTAATTYG-3' \\
& R: 5'-CACCRCRAATAAAATACRAAC-3' \\
TFPI2 & \\
Inside & M: 5'-TGGCGAAGTTGTTATTAGTC-3' \\
& U: 5'-TTGGTGAAGTTGTTATTAGTT-3' \\
Outside & F: 5'-ATTTTTTGTAGAAAGYGAGATG-3' \\
& R: 5'-AATACACRCAAAACTACCRC-3' \\
OPCML & \\
Inside & M: 5'-ACCGCTAAACGTAACGTCCG-3' \\
& U: 5'-ACCACTAAACATAACATCCA-3' \\
Outside & F: 5'-AAAAGTTTTAAGGYGGGGAY-3' \\
& R: 5'-TCRAAACTACTCCCRAAAAA-3',
\end{tabular}


Application of Multiplex Nested Methylated Specific PCR in Early Diagnosis of Epithelial Ovarian Cancer

on the electrodes to make the composites shining and the luminous intensity was measured with photoelectric multiplier, after which the concentration was calculated by 2-point adjusted calibration curve automatically. Serum CA125 was considered to be positive if its concentration was $\geq 35 \mathrm{U} / \mathrm{mL}$.

\section{Evaluation criteria}

The results of multiplex nested methylated specific PCR concluded that with human ovarian carcinoma cell line HO8910 as the positive control, the light stripe was regarded to be positive if its gray level was higher than $10 \%$ of positive control; to be positive if there was positive stripe in M system; to be negative if there was no positive stripe in $\mathrm{M}$ system but in $\mathrm{U}$ system; and to be invalid if there was no positive stripe in both $\mathrm{M}$ and $\mathrm{U}$ systems, which was excluded from the record.

\section{Statistical data analysis}

SAS9.3 statistical software was applied for all data analysis. Enumeration data was expressed by percentage (\%) and detected by $\chi 2$ among groups. Measurement data incongruent with normal distribution was expressed by median value and detected with Wilcoxon test. $p>0.05$ was considered to be statistically significant.

\section{Results}

Verification of multiple-primer nested methylated specific PCR system cells and sample methylation conditions

Single detection of the methylation conditions of RUNX3, TFPI2 and OPCML genes were conducted and the results demonstrated that in HO8910 cell lines, OPCML showed complete methylation (Figure 1), which was consistent with the results after the detection of the methylation conditions of above 3 genes simultaneously by the multiplex nested methylated specific PCR (Figure 2). The methylation spectrum of serum free DNA in EOC patients was completely contained in the DNA methylation spectrum of cancerous tissue (Figure 3 ).

Comparison of CA125 and methylation levels in control group, benign cancer group and EOC group

Serum CA125 and methylation levels in EOC group were evidently higher than those in benign group and control group $(p>0.05)$ (Figure 4). Serum CA125 level was apparently lower in patients with early EOC than in those with advanced EOC $(p>0.05)$, but there was no significant difference in serum free DNA methylation level $(p<0.05)$ (Figure 5).

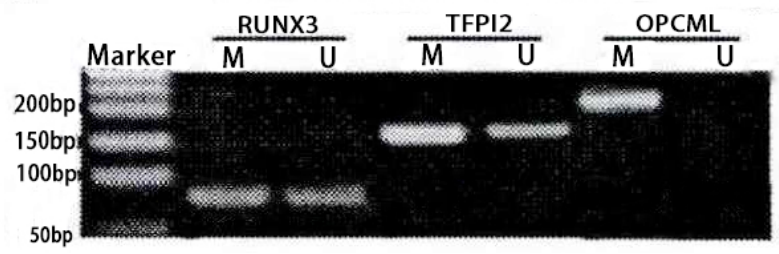

Figure 1. Methylation Conditions of RUNX3, TFPI2 and OPCML Genes in HO8910 Cell Lines by Single Gene Detection
Comparison of sensitivity, specificity and positive predicative value (PPV) of EOC detected with CA125 and multiplex nested methylated specific PCR

The sensitivity, specificity and PPV of EOC detected by the multiplex nested methylated specific PCR were markedly higher than those detected with CA125 ( $p>0.05)$.

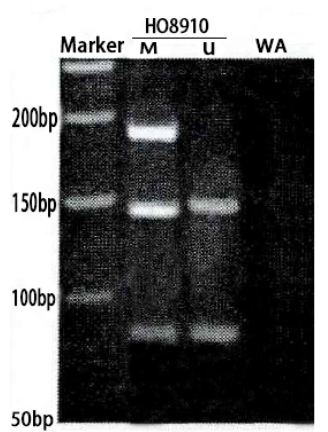

Figure 2. Methylation Conditions of RUNX3, TFPI2 and OPCML Genes in HO8910 Cell Lines detected Simultaneously by Multiplex Nested Methylated Specific PCR

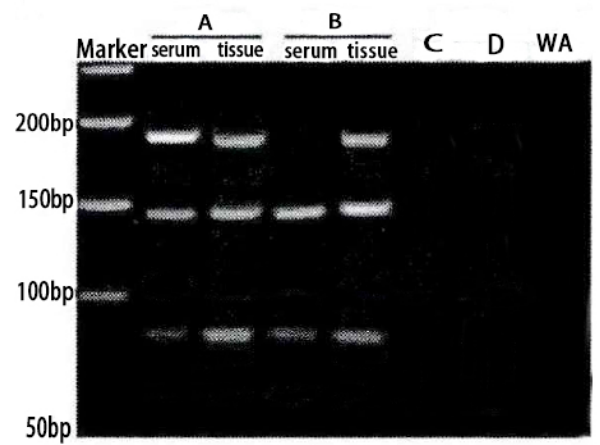

Figure 3. Methylated Conditions of RUNX3, TFPI2 and OPCML Genes in Control Group, Benign Cancer Group and EOC Group. Shows electrophoretic stripe in $\mathrm{m}$ system after multiplex nested methylated specific PCR
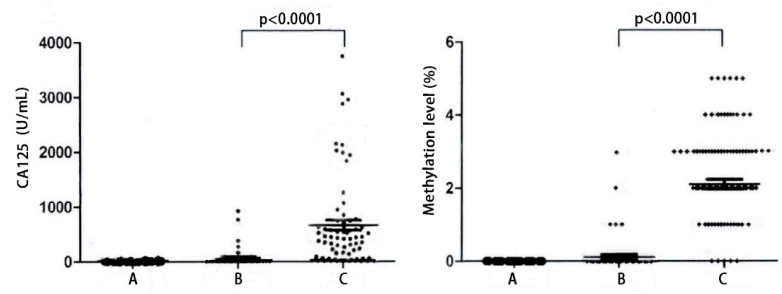

Figure 4. Comparison of CA125 and Methylation Levels in Control Group, Benign Cancer Group and EOC Group. A) Control group; B) Benign cancer group; C) EOC group
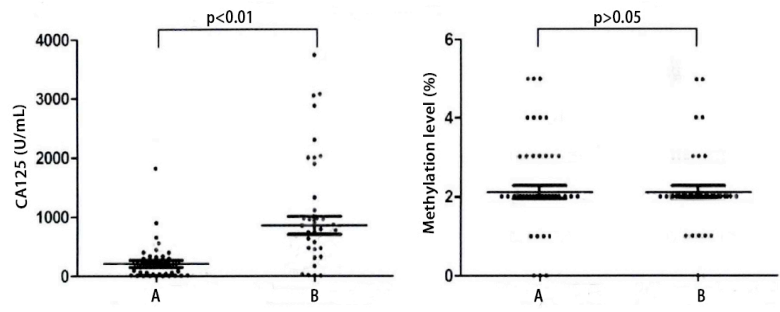

Figure 5. Comparison of CA125 and Methylation Levels in Early and Advanced EOC Patients. A) EOC patients in FIGO phase I II; B) EOC patients in FIGO phase III IV 
Table 2. Comparison of Sensitivity, Specificity and PPV of Two Detection Methods

\begin{tabular}{|c|c|c|c|}
\hline & Sensitivity & Specificity & PPV \\
\hline \multicolumn{4}{|c|}{ All patients with EOC } \\
\hline CA125 & $\begin{array}{l}73.24 \% \\
(52 / 71)\end{array}$ & $\begin{array}{r}63.41 \% \\
(78 / 123)\end{array}$ & $\begin{array}{l}53.61 \% \\
(52 / 97)\end{array}$ \\
\hline Methylation & $\begin{array}{r}90.14 \% \\
(64 / 71)\end{array}$ & $\begin{array}{r}91.06 \% \\
(112 / 123)\end{array}$ & $\begin{array}{l}85.33 \% \\
(64 / 75)\end{array}$ \\
\hline$\chi^{2}$ & 6.7798 & 26.7271 & 19.3875 \\
\hline $\mathrm{P}$ & 0.0092 & $<0.0001$ & $<0.0001$ \\
\hline \multicolumn{4}{|c|}{ Patients with early EOC } \\
\hline CA125 & $\begin{array}{l}53.85 \% \\
(21 / 39)\end{array}$ & - & $\begin{array}{l}31.82 \% \\
(21 / 66)\end{array}$ \\
\hline Methylation & $\begin{array}{l}84.62 \% \\
(33 / 39)\end{array}$ & - & $\begin{array}{l}75.00 \% \\
(33 / 44)\end{array}$ \\
\hline$\chi^{2}$ & 8.6667 & - & 19.6974 \\
\hline $\mathrm{P}$ & 0.0032 & - & $<0.0001$ \\
\hline \multicolumn{4}{|c|}{ Patients with advanced EOC } \\
\hline CA125 & $\begin{array}{r}84.38 \% \\
(27 / 32)\end{array}$ & - & $\begin{array}{l}37.50 \% \\
(27 / 72)\end{array}$ \\
\hline Methylation & $\begin{array}{r}93.75 \% \\
(30 / 32)\end{array}$ & - & $\begin{array}{l}73.17 \% \\
(30 / 41)\end{array}$ \\
\hline$\chi^{2}$ & 1.4436 & - & 13.2971 \\
\hline $\mathrm{P}$ & 0.2296 & - & 0.0003 \\
\hline
\end{tabular}

As to patients with advanced EOC, the PPV of CA125 was obviously lower than that detected by the multiplex nested methylated specific PCR $(p>0.05)$, but there was no significant difference in sensitivity $(p<0.05)$. And as to patients with early EOC, the 3 indexes detected by multiplex nested methylated specific PCR were apparently higher than those detected with CA125 ( $p>0.05)$ (Table 2).

\section{Discussion}

Ovarian carcinoma is dormant at onset, and $85 \%$ of patients are in advanced stage when clinically diagnosed, whose 5-year survival rate was $>45 \%$. However, the 5-year survival rate of patients with early ovarian carcinoma is up to $93 \%$, but accounting for only $15 \%$ of all patients with ovarian carcinoma (Australian Government, 2011). Therefore, early diagnosis is of great significance in improving the prognosis and reducing the mortality of patients with ovarian carcinoma. At present, CA125 is commonly used in clinic as a marker for ovarian carcinoma, but its sensitivity, specificity and PPV are relevantly low (McIntosh et al., 2007). Some scholars believed that the application of serum CA125 detection concomitant with transvaginal ultrasound probing could improve the diagnostic efficacy (Ji et al., 2014), but the results of the screening experiment of ovarian carcinoma, prostate carcinoma, colon cancer and lung cancer found that they could not reduce the mortality of patients with ovarian carcinoma (Buys et al., 2011).

DNA methylation is defined as the transformation of methyl to the 5th carbon atoms on cytosine under the catalysis of enzymes to form 5-methylcytosine so as to change the DNA construction and inhibit the gene transcription, thus leading to the inactivation of gene expressions (Tost et al., 2010). The enzyme used to catalyze DNA methylation is DNA methyltransference (DNMTs) that included DNMT1, DNMT3a, DNMT3b and DNMT31, in which DNMT1, as the persistent one, mainly participate in the maintenance of methylation condition, whereas DNMT3a and DNMT3b, as the de nove methyltransferences, can methylate $\mathrm{CpG}$ (Nelissen et al., 2011). DNA methylation has been widely used for the early diagnosis of malignant tumors such as leukemia, non-small cell lung cancer and renal cell carcinoma (Nordlund et al., 2015; Guo et al., 2015; Karami et al., 2015). Some scholars pointed out that the methylation levels of OPCML and SFRP1 genes in cancerous tissues, which had high specificity, sensitivity and accuracy in diagnosing cholangiocarcinoma, were potential molecular markers for the early diagnosis of cholangiocarcinoma (Amornpisutt et al., 2015). Chen et al detected the methylation levels of 7 tumor-inhibiting genes and the results revealed that the methylation levels of UHRF1-regulated SOCS3 and 3OST2 genes were closely associated with the development of endometrial carcinoma, which might become the important targets for the early diagnosis of endometrial carcinoma (Chen et al., 2014).

In clinical practices, tumor tissues are not always available, especially for patients with recurrent carcinoma. It was indicated in a study that the serum/plasma free DNA was evidently high in cancer patients than in healthy people, so these free DNA derived from the apoptotic and dead cells could be used to diagnose the tumors (Yu et al., 2013). Another research found that compared with healthy people, serum free DNAAlu methylation degree decreased more significantly in patients with esophagus carcinoma, which could be used as a potential biological marker for the diagnosis and treatment of nasopharyngeal carcinoma (Tiwawech et al., 2014). Additionally, in a study conducted by Yamashita et al, plasma CEA/CA19-9 level was detected together with plasma CDO1 methylation level and the results suggested that the combined detection was higher in detecting colorectal cancer than the single detection of CEA/CA19-9 level (Yamashita et al., 2014). As in the early diagnosis of ovarian carcinoma, it was reported that the detection rates of OPCML methylation in the serum and cancerous tissues of patients with ovarian carcinoma were $80.0 \%$ and $86.7 \%$, respectively, indicating that detecting serum free DNA OPCML methylation level could be an effective method for the diagnosis of ovarian carcinoma (Zhou et al., 2014). In addition, Liggett et al verified that the sensitivity and specificity of serum free DNA RASSF1A and PGR-PROX gene methylation were $80.0 \%$ and $73.3 \%$ respectively in identifying benign and malignant tumors (Liggett et al., 2011).

This study constructed multiplex nested methylated specific PCR system to detect the serum free DNA methylation condition, which suggested higher sensitivity and specificity in the detection of patients with early EOC, thus bringing about new thoughts for the early diagnosis of EOC. However, the target genes selected in this study also had relevantly high methylation rate in other malignant tumors, so the specificity was limited. In order to further improve the detection specificity, epigenetic sequencing of ovarian carcinoma could be performed to search new methylation gene with high specificity for patients with ovarian carcinoma. Additionally, whether 
this retrospectively analysis is feasible in clinic needs to be verified by further prospective studies.

\section{Acknowledgements}

This study was supported by Academician Workstation Construction Projects of Guangdong Province (2012B09050007); International Cooperation Projects of Guangdong Province (2010B050100022).

\section{References}

Amornpisutt R, Proungvitaya S, Jearanaikoon P, et al (2015). DNA methylation level of OPCML and SFRP1: a potential diagnostic biomarker of cholangiocarcinoma. Tumour Biol, (in press).

Buys SS, Partridge E, Black A, et al (2011). Effect of screening on ovarian cancer mortality: the prostate, lung, colorectal and ovarian (PLCO) cancer screening randomized controlled trial. JAMA, 305, 2295-303.

Clarke-Pearson DL (2009). Screening for ovarian cancer. New Engl J Med, 361, 170-7.

Chen H, Zhang C, Sheng Y, et al (2014). Frequent SOCS3 and 3OST2 promoter methylation and their epigenetic regulation in endometrial carcinoma. Am J Cancer Res, 5, 180-90.

Guo S, Yan F, Xu J, et al (2015). Identification and validation of the methylation biomarkers of non-small cell lung cancer (NSCLC). Clin Epigenetics, 7, 3.

Ji T, Zheng ZG, Wang FM, et al (2014). Differential microRNA expression by Solexa sequencing in the sera of ovarian cancer patients. Asian Pac J Cancer Prev, 15, 1739-43.

Karami S, Andreotti G, Liao LM, et al (2015). LINE-1 methylation levels in pre-diagnostic leukocyte DNA and future renal cell carcinoma risk. Epigenetics, (in press).

Liggett TE, Melnikov A, Yi Q, et al (2011). Distinctive DNA methylation patterns of cell-free plasma DNA in women with malignant ovarian tumors. Gynecol Oncol, 120, 113-20.

McIntosh MW, Liu Y, Drescher C, et al (2007). Validation and characterization of human kallikrein 11 as a serum marker for diagnosis of ovarian carcinoma. Clin Cancer Res, 13, 4422-8.

Nelissen EC, van Montfoort AP, Dumoulin JC, et al (2011). Epigenetics and the placenta. Hum Reprod Update, 17, 397-417.

Nordlund J, Bäcklin CL, Zachariadis V, et al (2015). DNA methylation-based subtype prediction for pediatric acute lymphoblastic leukemia. Clin Epigenetics, 7, 11.

Pothuri B, Leitao MM, Levine DA, et al (2010). Genetic analysis of the early natural history of epithelial ovarian carcinoma. PLoS One, 5, 10358

Shih IeM, Kurman RJ (2004). Ovarian tumorigenesis: a proposed model based on morphological and molecular genetic analysis. Am J Pathol, 164, 1511-8.

Siegel R, Deepa Naishadham MA, Ahmedin Jemal DVM (2013). Cancer statistics, 2013. CA Cancer J Clin, 63, 11-30.

Tost J (2010). DNA methylation: an introduction to the biology and the disease-associated changes of a promising biomarker. Mol Biotechnol, 44, 71-81.

The Department of Health, Australian Government (2011). Cancer facts and figures. http://www.health.gov.au/internet/ main/publishing.nsf/Content/cancer-facts

Tiwawech D, Srisuttee R, Rattanatanyong P, et al (2014). Alu methylation in serum from patients with nasopharyngeal carcinoma. Asian Pac J Cancer Prev, 15, 9797-800.

Wang B, Liu SZ, Zheng RS, et al (2014).Time trends of ovarian cancer incidence in China. Asian Pac J Cancer Prev, 15,
191-3.

Yu Shaorong, Liu Baorui, Lu Jianwei, et al (2013). Circulating DNA as potential biomarker for cancer individualized therapy. J Int Transl Med, 1, 144-51.

Yamashita K, Waraya M, Kim MS, et al (2014). Detection of methylated CDO1 in plasma of colorectal cancer; a PCR study. PLoS One, 9, 113546.

Zhou F, Ma M, Tao G, et al (2014). Detection of circulating methylated opioid binding protein/cell adhesion moleculelike gene as a biomarker for ovarian carcinoma. Clin Lab, 60, 759-65. 